\title{
Expression of OsPSTOL1 Improves the Phosphorus Utilization Efficiency of Transgenic Populus tomentosa
}

\author{
Lu Litang ${ }^{1,2}$,Wang $\mathrm{Lu}^{1}$, Ying Yang ${ }^{1}$, Degang Zhao ${ }^{1}$ and Yao Xinzhuan ${ }^{2 *}$ \\ ${ }^{1}$ College of Life Sciences and The Key Laboratory of Plant Resource Conservation and Germplasm Innovation in Mountainous \\ Regions (Ministry of Education), Institute of Agro-Bioengineering, Guizhou University, Guiyang, 550025, China CC \\ ${ }^{2}$ College of Tea Science, Guizhou University, Guiyang, 550025, People's Republic of China \\ ${ }^{3}$ Guizhou Academy of Agricultural Science, Guiyang, 550006, China \\ *For correspondence: ltlv@gzu.edu.cn; 943973129@qq.com \\ Received 15 June 2020; Accepted 31 October 2020; Published 25 January 2021
}

\begin{abstract}
OsPSTOL1 encodes a phosphorus (P) deficiency-tolerant protein expressed in the aus rice variety, Kasalash, which functions by enhancing the ability of the plant to obtain $\mathrm{P}$ and other nutrients; however, its role in woody plants remains unknown. In the present study, we isolated OSPSTOL1 from Oryza sativa L. and subsequently generated OsPSTOL1-overexpressing transgenic Populus tomentosa. It was found that in the state of P deficiency, the transgenic P. tomentosa had a greater root length, the symptoms of P-deficiency appeared much later, and the total nitrogen (TN) and phosphorus (TP) content was higher as compared with that of wild type plants. Upregulation of P-tolerance genes in transgenic P. tomentosa under P-deficient conditions was analyzed by real-time quantitative reverse transcription polymerase chain reaction (RT-qPCR), the results of which were consistent with the physiological traits. In summary, these findings show that OsPSTOL1 plays a positive role in the regulation of $\mathrm{P}$ uptake and utilization in transgenic $P$. tomentosa trees under P-deficient conditions and provide evidence for the potential application of OSPSTOL1 in woody plants to overcome soil P deficiency. (C) 2021 Friends Science Publishers
\end{abstract}

Keywords: OsPSTOL1; Nitrogen and phosphorus content; Phosphorus utilization efficiency; Populus tomentosa Abbreviations: P (phosphorus); Pi (inorganic phosphate); ATP (adenosine triphosphate); OsPSTOL1 (phosphate starvation tolerance 1); MS (Murashige and Skoog); GUS ( $\beta$-glucuronidase); PHO1 (phosphorus 1); PHT1 (phosphate transporters 1); PHR1 (phosphate starvation response 1)

\section{Introduction}

Phosphorus (P) is an essential element for life in natural ecosystems, especially for plant growth. Inorganic phosphate (Pi) derivatives are also involved in almost all significant metabolic pathways, in addition to being present as a structural component of many biochemicals including nucleic acids, coenzymes, phosphoproteins, and phospholipids (Xiang et al. 2016; Milner et al. 2018). Moreover, $\mathrm{P}$ is also the key component of the cellular energy source, adenosine triphosphate (ATP), which plays an important role in protein and fat metabolism. The concentration of $\mathrm{P}$ in the vacuole of plant cells has a buffering effect on the cytoplasm and maintains normal photosynthesis of the leaves to a certain extent. $\mathrm{P}$ is mainly absorbed by plants from soil in the form of orthophosphate (hydrogen phosphate, $\mathrm{Na}_{2} \mathrm{HPO}_{4}$ or dihydrogen phosphate, $\mathrm{NaH}_{2} \mathrm{PO}_{4}$ ) (Irfan et al. 2020). $\mathrm{P}$ deficiency affects a series of cellular metabolic processes, such as carbon metabolism and photosynthesis (Rao and Pessarakli 1996), resulting in stagnant growth, short and thin plants, small leaves, delayed maturity, small fruit or empty seeds, and reduced yield and quality (Yu et al. 2010).

According to statistical data, $40-60 \%$ of the world's arable land lacks sufficient available $\mathrm{P}$, which results in a decline in crop yields (Cheng et al. 2011); globally, 5.7 billion hectares of land are deficient in $\mathrm{P}$ (Jewell et al. 2010). In addition, the price of Pi fertilizer is relatively expensive, and the annual global $\mathrm{P}$ consumption has increased by approximately 3\% (Gaxiola et al. 2011), with roughly 40 million tons of $\mathrm{Pi}$ fertilizer currently being applied. Surprisingly, less than $20 \%$ of $\mathrm{Pi}$ is absorbed by crops and the rest is wasted, resulting in poor utilization of soil $\mathrm{P}$ and requiring increased $\mathrm{Pi}$ fertilizer application at additional costs. To cope with low-pressure stress, plants have evolved many mechanisms such as changing the anatomical structure and morphology of the roots, symbiosis, and biochemical changes in metabolites (Ceasar 2018; Maharajan et al. 2018). Many plant species are known to grow in low-P soils, such as certain tropical plants, legumes, and corn, achieving a moderately successful yield (Smith

To cite this paper: Litang L, W Lu, Y Yang, D Zhao, Y Xinzhuan (2021). Expression of OsPSTOL1 improves the phosphorus utilization efficiency of transgenic Populus tomentosa. Intl J Agric Biol 25:651-658 
1934; Tong et al. 2001); however, whether woody plants can tolerate P-deficiency remains unknown. A quantitative trait locus (QTL) for phosphate starvation tolerance 1 (OsPSTOL1) has been localized to chromosome 12 in rice and is known to be involved in P uptake (Ni and Wand 1998; Pariasca-Tanaka et al. 2014). OsPSTOL1 is considered to have the potential to improve yield in P-deficient and/or drought-prone environments across diverse genetic backgrounds (Chin et al. 2010; Gamuyao et al. 2012). At present, studies on OSPSTOL1 are limited to crops, and its role in woody plants remains to be elucidated.

Plants exhibit great developmental plasticity in response to adverse environments. A large number of studies have confirmed that the morphological distribution of roots directly affects the absorption of nutrients and water by plants, which in turn influences the growth and ecological functions of their above-ground parts (Liang et al. 2007). Therefore, under P-deficient conditions, the development of the root system improves plant nutrient absorption to a certain extent (Qizhen et al. 2008).

In the present study, we investigated the role of OsPSTOL1 in $\mathrm{P}$ absorption and utilization in P. tomentosa and found that overexpression of OSPSTOL1 increased the absorption of $\mathrm{P}$ under low-P conditions. Our experimental results provide a reference for the further exploration of OsPSTOL1 gene function, molecular mechanism, and effect on plant growth and development, in addition to providing a basis for the adaptation of woody plants to poor growth with a view to achieving overall improvement in wood production.

\section{Materials and Methods}

\section{Experimental materials}

P. tomentosa seedlings were preserved by the Agricultural Bioengineering Research Institute, Guiyang Guizhou, China. The SYBR® Select Master Mix, Murashige and Skoog Medium, rifampicin, kanamycin, timentin, sucrose, agar, and GUS stain were obtained from Beijing huayueyang.biomart.cn.

\section{Cloning of OSPSTOLI and construction of plant expression vectors}

OsPSTOL1 cDNA was used as a template for PCR with the following primers: $\mathrm{F}$ : 5'AGAGCTCTGATCTATGAGTACATGC-3'; R: 5'CAGTCTATCCCAGCTCAGGG-3'. The PCR product was cloned into the T-vector (Beijing Biomed Biotechnology Co., Ltd.) and sequenced (Wuhan Jinkairui Biological Engineering Co., Ltd. China). The pSH737 plasmid was restriction digested with EcoRI and XbaI, and the OsPSTOL1 sequence was cut with KpnI and BamHI. The vector contained the OSPSTOL1 expression element, with the $35 \mathrm{~S}$ promoter driving the GUS-NPTII fusion protein as a screening marker and reporter. The PCR product size of OsPSTOLl is 119 bp (Fig. 1).

\section{Plant transformation and transgenic plant detection}

P. tomentosa was transformed with OSPSTOL1 using the Agrobacterium-mediated leaf disc method. Agrobacteria expressing pSH737-35S-OsPSTOL1 were resuspended in Murashige and Skoog (MS) nutrient medium and the concentration was measured using a nucleic acid analyzer at $\mathrm{OD}_{600}(0.4)$. The $P$. tomentosa leaf disks were subjected to the Agrobacterium solution for 6-8 min and dried on sterile filter paper prior to being placed on co-cultivation medium (MS + $0.1 \mathrm{mg} \mathrm{L}^{-1} \mathrm{NAA}$ ) for $2-3 \mathrm{~d}$ at $26 \pm 2{ }^{\circ} \mathrm{C}$ in the dark. Next, the leaves were transferred sequentially onto callus induction medium $\left(\mathrm{MS}+2.0 \mathrm{mg} \mathrm{L}^{-1} \mathrm{ZT}+1.0 \mathrm{mg} \mathrm{L}^{-1} \mathrm{NAA}+\right.$ $200 \mathrm{mg} \mathrm{L}^{-1}$ timentin $+50 \mathrm{mg} \mathrm{L}^{-1}$ kanamycin $+30 \mathrm{~g} \mathrm{~L}^{-1}$ sugar $+8 \mathrm{~g} \mathrm{~L}^{-1}$ agar powder $\mathrm{pH} 5.80$ ); value-added medium (MS + $1.0 \mathrm{mg} \mathrm{L^{-1 }} 6-\mathrm{BA}+200 \mathrm{mg} \mathrm{L}^{-1}$ timentin $+50 \mathrm{mg} \mathrm{L}^{-1}$ kanamycin $+30 \mathrm{~g} \mathrm{~L}^{-1}$ sugar $+8 \mathrm{~g} \mathrm{~L}^{-1}$ agar powder $\mathrm{pH} 5.80$ ); and bud induction medium (MS + $2.0 \mathrm{mg} \mathrm{L}^{-1} \mathrm{ZT}+1.0 \mathrm{mg} \mathrm{L}$ ${ }^{1} \mathrm{NAA}+200 \mathrm{mg} \mathrm{L}^{-1}$ timentin $+50 \mathrm{mg} \mathrm{L}^{-1}$ kanamycin $+30 \mathrm{~g}$ $\mathrm{L}^{-1}$ sugar $+8 \mathrm{~g} \mathrm{~L}^{-1}$ agar powder $\mathrm{pH}$ 5.80) (Dai 2018; Yao et al. 2020). Following shoot development at $3-5 \mathrm{~cm}$, the shoots were separated from the calli and transferred onto rooting medium $\left(\mathrm{MS}+0.1 \mathrm{mg} \mathrm{L}^{-1} \mathrm{NAA}+200 \mathrm{mg} \mathrm{L}^{-1}\right.$ timentin +50 $\mathrm{mg} \mathrm{L}^{-1}$ kanamycin $+30 \mathrm{~g} \mathrm{~L}^{-1}$ sugar $+6.5 \mathrm{~g} \mathrm{~L}^{-1}$ agar powder $\mathrm{pH}$ 5.80). The rooted shoots were subsequently transplanted into pots and allowed to grow. Two or three leaves from each plant were analyzed by $\beta$-glucuronidase (GUS) staining and PCR to verify OSPSTOL1 gene integration.

\section{Plant growth conditions}

$\mathrm{T}_{0}$ generation OSPSTOL1-overexpressing transgenic $P$. tomentosa strains were cultured to rooting in MS medium (under a $16 \mathrm{~h}$ light/8 $\mathrm{h}$ dark photoperiod with photosynthetically active radiation of $200 \mu \mathrm{mol} \mathrm{m}{ }^{-2} \mathrm{~s}^{-1}$ ) and then transplanted into pots containing medium-sized $(2-4$ $\mathrm{mm}$ diameter) vermiculite. The plants were cultured for 3-4 weeks prior to the experiment (MS full-nutrition solution).

\section{Phosphorus deficiency experiment}

P-deficient conditions were introduced by transferring the transgenic and wild type $P$. tomentosa seedlings to Hoagland's P-deficient culture medium (1/4 of the $\mathrm{P}$ content) and growing them in a greenhouse (under a 16 $\mathrm{h}$ light $/ 8 \mathrm{~h}$ dark photoperiod, $25^{\circ} \mathrm{C}$, and $50 \%$ relative humidity) until symptoms of $\mathrm{P}$ deficiency developed. Each experiment was repeated at least three times, the results of which were similar.

\section{Quantitative reverse transcription PCR (RT-qPCR)}

Synthesis of cDNA from total RNA $(10 \mu \mathrm{L})$ (fresh leaves of 
wild type and transgenic lines) was conducted using an MMLV reverse transcriptase kit (28025013, Invitrogen ${ }^{\mathrm{TM}}$ ) according to the manufacturer's instructions. RT-qPCR was performed using the SYBR® Select Master Mix, CFX Manager (Bio-Rad), and CFX96TM RT PCR detection system (Bio-Rad). A three-step reaction procedure was performed in 96-well optical reaction plates. The cycling profile was $95^{\circ} \mathrm{C}$ for $15 \mathrm{~s} ; 39$ cycles of $95^{\circ} \mathrm{C}$ for $10 \mathrm{~s}, 55^{\circ} \mathrm{C}$ for $30 \mathrm{~s}$, and $72^{\circ} \mathrm{C}$ for $30 \mathrm{~s}$; and the solubility curve was $95^{\circ} \mathrm{C}$ for $15 \mathrm{~s}, 60^{\circ} \mathrm{C}$ for $60 \mathrm{~s}, 95^{\circ} \mathrm{C}$ for $15 \mathrm{~s}$, and $60^{\circ} \mathrm{C}$ for 15 $\mathrm{s}$. The total volume of the PCR reaction $(25 \mu \mathrm{L})$ contained double-distilled $\mathrm{H}_{2} \mathrm{O}(8.7 \mu \mathrm{L})$; forward $(0.90 \mu \mathrm{L})$ and reverse $(0.90 \mu \mathrm{L})$ primers; SYBR® Select Master Mix $(12.5 \mu \mathrm{L})$; and cDNA $(2.0 \mu \mathrm{L}, 0.16 \mu \mathrm{g})$. The $2^{-\Delta \Delta \mathrm{Ct}}$ method was used to calculate the relative gene expression (Wu et al. 2016). The primer sequences of related genes are shown in Table 1.

\section{Determination of total $N, P$ and $K$ content}

Each plant sample (3-5 leaves from the base to the top) was pulverized, passed through a $0.2-\mathrm{mm}$ sieve, and dried at $60-80^{\circ} \mathrm{C}$.

Determination of $\mathbf{N}$ content: A mass of $0.10-0.20 \mathrm{~g}$ ground and dried plant sample $(0.25-0.5-\mathrm{mm}$ sieve) was weighed and placed in a $100-\mathrm{mL}$ Erlenmeyer flask. The sample was moistened with a small amount of water, to which $5 \mathrm{~mL}$ concentrated sulfuric acid was added, covered, and shaken overnight. The neck of the flask was slowly heated, and as the temperature gradually increased, the concentrated sulfuric acid was decomposed, emitting a white smoke. The sample was digested until the solution was evenly brownishblack in color, to which 10 drops of $\mathrm{H}_{2} \mathrm{O}_{2}$ were added while shaking. The sample was reheated to almost boiling for 10-20 min and allowed to cool slightly, following which 5-10 drops of $\mathrm{H}_{2} \mathrm{O}$ were added. This step was repeated 2-3 times until the liquid became colorless, which was then heated for 5-10 min (to drive off the $\mathrm{H}_{2} \mathrm{O}_{2}$ ). The sample was allowed to cool, the flask was rinsed with water, and the liquid was transferred to a volumetric flask containing a constant volume of water and subsequently filtered.

A 5-10-mL aliquot of the clear liquid was transferred to a distillation tube, which was placed in the nitrogen analyzer. A volume of $2 \mathrm{~mL}$ boric acid was placed in a 150$\mathrm{mL}$ Erlenmeyer flask, to which 2 drops of indicator were added and shaken. The Erlenmeyer flask was placed in the nitrogen analyzer to condense. At the end of the tube, where the mouth of the tube is $3-4 \mathrm{~cm}$ above the liquid level of boric acid, approximately $5 \mathrm{~mL} 10 \mathrm{~mol} \cdot \mathrm{L} \mathrm{NaOH}$ solution was added to the distillation tube and subjected to steam distillation. When the level of the distillate was roughly 50 $\mathrm{cm}$, the results were calculated.

Determination of $\mathbf{P}$ content: A $10-\mathrm{mL}$ aliquot of the test solution that had been digested and boiled at a constant volume (same as total $\mathrm{N}$ determination) was placed in a 50$\mathrm{mL}$ volumetric flask, to which 2 drops of 2,6 dinitrophenol indicator were added, and the $\mathrm{pH}$ was adjusted with 6 $\mathrm{mo} 1 \cdot \mathrm{L}^{-1} \mathrm{NaOH}$ to a yellow color. Subsequently, $10 \mathrm{~mL}$ ammonium vanadyl molybdate reagent was added and the volume made constant with water. The flask was shaken well and incubated for $5 \mathrm{~min}$, following which the absorbance was measured using a spectrophotometer at 450 $\mathrm{nm}$. The zero point of the instrument was adjusted with a blank solution. The standard curve was prepared using 0 , $1.0,2.5,5,7.5,10.0$, and $15.0 \mu \mathrm{g} \cdot \mathrm{mL}^{-1} \mathrm{P}$. The results were subsequently calculated.

Determination of $\mathbf{K}$ content: A 5-10-mL aliquot of the test solution that had been digested and boiled at a constant volume (same as total $\mathrm{N}$ determination) was placed in a 50 $\mathrm{mL}$ volumetric flask and made up to a constant volume with water. The sample was measured directly using a flame photometer, and the galvanometer reading was recorded. The mass fraction (\%) of quasi-plant potassium was calculated according to the calibration curve or linear regression equation.

\section{Statistical analysis}

Three independent biological replicates and three technical replicates were used to calculate the mean value and standard deviation (SD) of the metabolite concentrations. The SPSS 17.0 software was used to determine significant differences by Duncan's multiple-range test. Any difference relative to the control at $* P<0.05$ or $* * P<0.01$ was considered statistically significant.

\section{Results}

\section{Generation of genetically modified $P$. tomentosa}

The leaves and stems of sterile $P$. tomentosa seedlings were used as explants to genetically transform $P$. tomentosa via Agrobacterium-mediated leaf disc transformation. Kanamycin (Kan) was added to the medium for selection. Following refinement of resistant plants for approximately 7 days (during this period, the leaves should not lose too much water), the root medium was removed and the plants were planted in the greenhouse soil for cultivation. A few young leaves were taken for $\beta$-glucosidase (GUS) histochemical staining, and genomic DNA was removed from the plants for PCR verification. We obtained 12 transgenic $P$. tomentosa plants (Fig. 2), of which 3 overexpressing lines, along with 3 wild type strains, were selected for subsequent experiments $(5,8,13)$.

\section{Expression of OsPSTOL1 in transgenic $P$. tomentosa regulates their growth and development}

To verify whether OSPSTOL1 influences the $\mathrm{P}$ uptake capacity of $P$. tomentosa, we subjected wild type and transgenic plants to P-deficient conditions and observed their growth status in real time. Prior to the introduction of P-deficient conditions, the above-ground development of 
Lu et al. / Intl J Agric Biol, Vol 25, No 3, 2021

Table 1: Oligonucleotide primers used for confirmation of transgenic lines and expression analysis of various endogenous genes

\begin{tabular}{|c|c|c|c|c|}
\hline Gene name & Gene ID & Annealing $\operatorname{Tm}\left({ }^{\circ} \mathrm{C}\right)$ & Product size (bp) & Primer (5'-3') \\
\hline$\overline{P H T 1}$ & Potri.010G072000.1 & 55 & 115 & $\begin{array}{l}\text { TGCTGGGCTTACATTCTATTGG } \\
\text { TCTGACGCTGCTTGTATTGC }\end{array}$ \\
\hline PHT2 & Potri.010G046300.1 & 55 & 120 & $\begin{array}{l}\text { GAGGAACATCAAGAAACGGTAA } \\
\text { CAAGCCCTGTCCCAAAGTC }\end{array}$ \\
\hline PHR1 & Potri.002G257800.6 & 55 & 203 & $\begin{array}{l}\text { CTGCACTAGAAGGAGGATCACA } \\
\text { ATGGCTCAGAATATTCAGGAGT }\end{array}$ \\
\hline PHOI & Potri.008G110800.1 & 55 & 175 & $\begin{array}{l}\text { GTGCTAGGCGATGGTTTGAC } \\
\text { TCCTGCTGCTAACATTGCTGA }\end{array}$ \\
\hline Actin & Potri.001G309500 & 55 & 156 & $\begin{array}{l}\text { AAACTGTAATGGTCCTCCCTCCGG } \\
\text { CATCATCACAATCACTCTCCGA }\end{array}$ \\
\hline
\end{tabular}

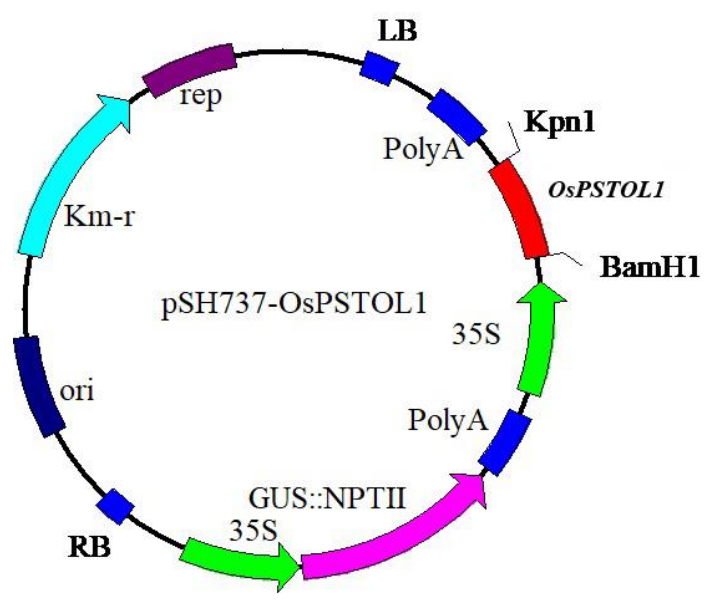

Fig. 1: pSH-35S-OsPSTOL1 expression plasmid map. RB: Right border of T-DNA; 35S: CaMV 35S poly A; LB: Left border of T-DNA; GUS: $\beta$-glucuronidase reporter gene

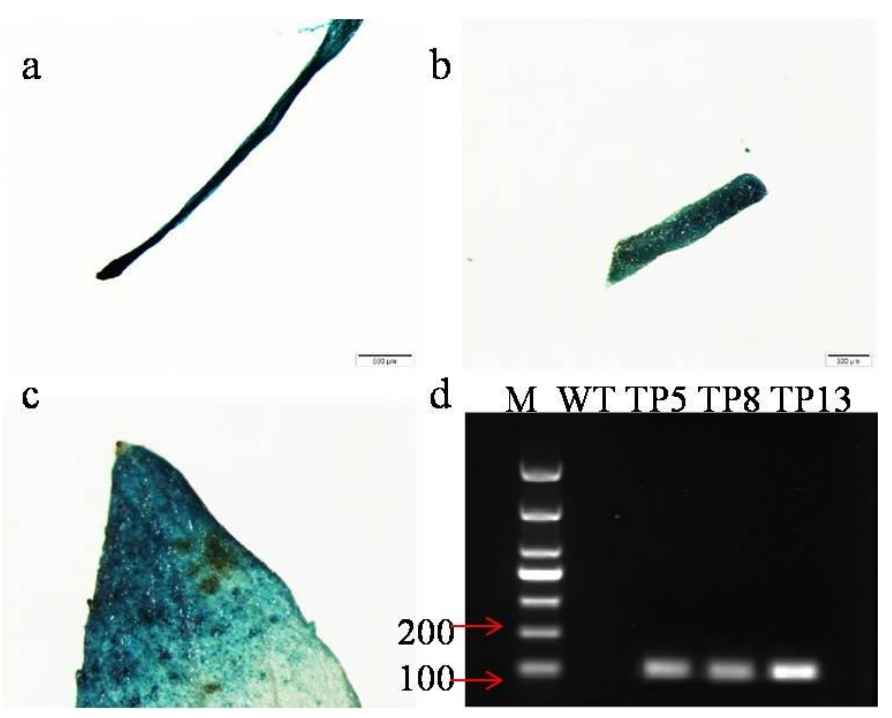

Fig. 2: $\beta$-Glucuronidase (GUS) staining and PCR identification. (a) GUS staining of the roots of transgenic P. tomentosa. (b) GUS staining of the stems of transgenic P. tomentosa. (c) GUS staining of the top leaves of transgenic P. tomentosa. (d) PCR identification of transgenic $P$. tomentosa (119 bp). Molecular identification of leaves extracted from the same part of wild type and transgenic $P$. tomentosa. M: DNA Marker; WT: wild type; TP: transgenic plant lines (TP5, TP8, TP13)

wild type and transgenic plants was almost identical (Fig. 3a). However, under P-deficient conditions, the leaves of wild type plants exhibited typical symptoms of $\mathrm{P}$ deficiency, such as chlorosis, yellowing, and tissue necrosis (Fig. 3c), while the leaves of transgenic plants were green (Fig. 3b). After being subjected to these conditions for 20 days, the root length and fresh weight of transgenic and wild type $P$. tomentosa were measured (Fig. 3b; Table 2). 
Table 2: Root fresh weight and length of wild type and transgenic P. tomentosa

\begin{tabular}{lll}
\hline Type & Root fresh weight $(\mathrm{g})$ & Root length $(\mathrm{cm})$ \\
\hline WT & $3.21 \pm 0.22$ & $17.55 \pm 1.11$ \\
TP & $4.58 \pm 0.163 *$ & $23.97 \pm 2.97 *$ \\
\hline $\begin{array}{l}\text { Note: Root length refers to the longest distance of the plant root. Data are the average of three wild type and three transgenic plants, respectively. Specific data for each strain are } \\
\text { shown in Supplementary Materials. TP (mean value for transgenic plants); *statistically significant }\end{array}$
\end{tabular}
shown in Supplementary Materials. TP (mean value for transgenic plants); *statistically significant

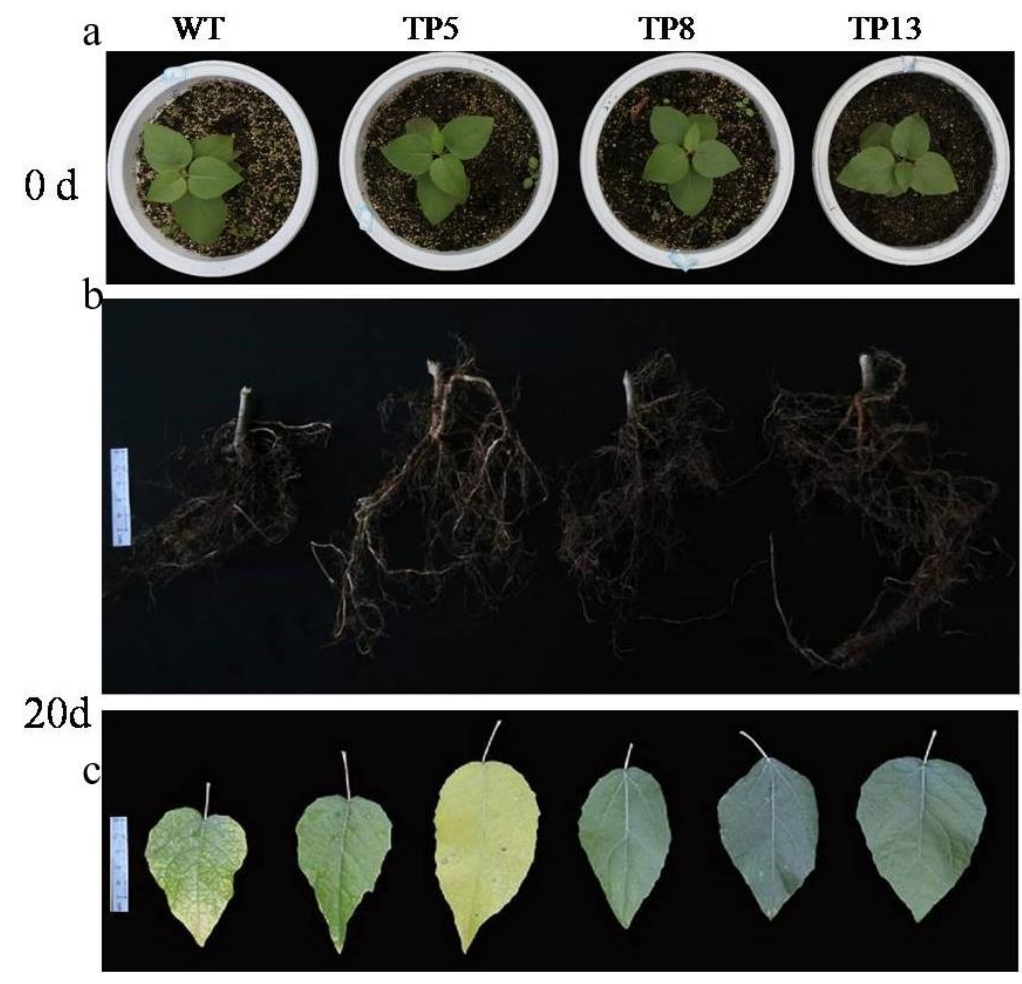

Fig. 3: Root growth and symptoms of transgenic and wild type (WT) $P$. tomentosa under P-deficient conditions. (a) Transgenic and WT $P$. tomentosa prior to the introduction of P-deficient conditions. (b) Roots of transgenic and WT P. tomentosa following the introduction of P-deficient conditions for $20 \mathrm{~d}$. (c) Transgenic and WT P. tomentosa following the introduction of P-deficient conditions for $20 \mathrm{~d}$. The order of the leaves from left to right is WT1, WT2, WT3, TP5, TP8, and TP13. The displayed leaves are fifth from the base

\section{Effects of OsPSTOLI on the total N, P and $\mathrm{K}$ content of transgenic $P$. tomentosa}

We investigated whether OSPSTOL1 can regulate the total $\mathrm{N}$, $\mathrm{P}$, and $\mathrm{K}$ content of wild type and transgenic $P$. tomentosa under low-P conditions (Fig. $4 \mathrm{a}-\mathrm{c}$ ) and found that the $\mathrm{N}$ and $\mathrm{P}$ content of transgenic plants was 1.18- and 1.35 -fold higher than that of wild type plants, respectively. However, there was no obvious difference in $\mathrm{K}$ content between wild type and transgenic plants.

\section{OsPSTOL1 influences the expression of related genes}

Plants have evolved to possess many mechanisms for adapting to low $\mathrm{P}$ concentrations, including the induction of high-affinity $\mathrm{P}$ transporters, acid phosphatase, and organic acid secretion (Wasaki et al. 2006). To investigate the mechanism of OsPSTOL1 underlying tolerance to $\mathrm{P}$ deficiency, we examined several typical stress response genes, including Phosphorus 1 (PHOl), Phosphate transporter 1 ( $P H T 1)$, and Phosphate starvation response 1 (PHRl). The expression levels of these genes under Pdeficient conditions were detected by RT-qPCR, and it was found that their expression levels were higher in transgenic plants as compared with those in wild type plants (Fig. 5). These results indicate that OSPSTOL1 plays a significant role in regulating the expression of $\mathrm{P}$ transporter- and acid phosphatase-related genes in $P$. tomentosa.

\section{Discussion}

$\mathrm{P}$ is an essential macronutrient for plant growth and development. $\mathrm{Pi}$ is the major form of $\mathrm{P}$ that the plants absorb and assimilate, which is taken up by the roots and translocated between cells or tissues via the action of $\mathrm{Pi}$ transporters. When the surrounding environment is nutrientdeficient, the growth of the plant will be directly affected. In addition to the height of the plant, size of the leaves, and growth of the roots, the dry weight of the plant can also serve as a measure of the N, P and K content (Sheng 1999). 

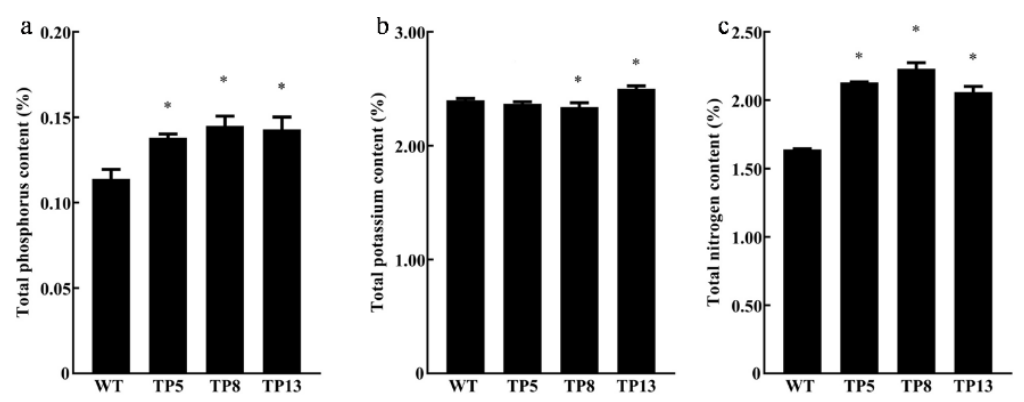

Fig. 4: Nitrogen (N), P, and potassium (K) content of transgenic and wild type (WT) P. tomentosa following the introduction of Pdeficient conditions. (a) P content of transgenic and WT P. tomentosa following the introduction of P-deficient conditions. (b) K content of transgenic and WT P. tomentosa following the introduction of P-deficient conditions. (c) $\mathrm{N}$ content of transgenic and WT P. tomentosa following the introduction of P-deficient conditions. Three WT samples were mixed

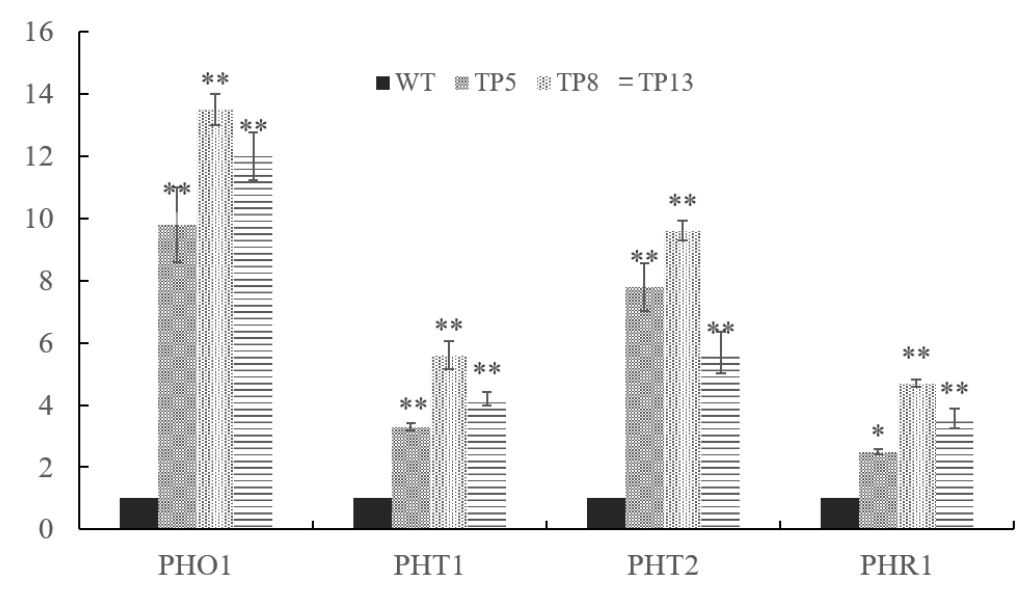

Fig. 5: Expression pattern of the typical stress response genes, PHT1, PHT2, PHR1, and PHO1, in P. tomentosa following the introduction of P-deficient conditions, as assessed by RT-qPCR. WT (three wild type samples mixed); TP (three transgenic samples mixed). Data are representative of at least three independent experiments

The state of $\mathrm{P}$ deficiency in the soil is not only important for crop yield but also for the wood yield of woody plants, since this is also limited in low-P soil (Jia et al. 2017).

OSPSTOL1 has previously been cloned and its function in rice verified (Heuer et al. 2009). This gene has been shown to be directly involved in the uptake and utilization of $\mathrm{P}$ in rice (Chin et al. 2011). In P-deficient soils, overexpression of OSPSTOL1 in crops can encourage normal growth and increase yields, which is of great significance for increasing food production; however, OSPSTOL1 remains functionally uncharacterized in woody plants. Here, to further investigate its function, we isolated OSPSTOL1 from rice and demonstrated a positive effect on the uptake and utilization of $\mathrm{P}$ in transgenic $P$. tomentosa.

The present study found that under P-deficient conditions, the associated symptoms in transgenic P. tomentosa were significantly delayed as compared with those in the wild type plant (Fig. 3c). Malnutrition during the growth and development of plants is directly manifested as the health of the leaves, for example: fading; a color change from green to yellow, red, or purple; tissue necrosis, black heart, dead spots, atrophy or death of growth points; abnormal plant type; organ deformities; and delayed growth or progression. Moreover, the $\mathrm{N}, \mathrm{P}$, and $\mathrm{K}$ content of the transgenic and wild type $P$. tomentosa was also measured under low-P conditions, and the results show that the $\mathrm{N}$ and $\mathrm{P}$ content of transgenic $P$. tomentosa was higher than that of the wild type plant; however, the $\mathrm{K}$ content was not significantly different between the two plants. Taken together, these data suggest that OsPSTOL1 participates in and improves the regulation of $\mathrm{P}$ absorption and utilization in plants under P-deficient conditions and reduces the associated symptoms. Studies have shown that low $\mathrm{P}$ concentrations inhibit the elongation of primary roots and promote the differentiation of lateral roots (Sánchez-Calderón et al. 2005). Plant roots absorb $\mathrm{P}$ nutrients from the soil solution, and the basic process of regulating $\mathrm{P}$ transport to vascular tissues is regulated by related genes (Schünmann et al. 2004). In the present study, it was found that under P-deficient conditions, the main roots of transgenic $P$. tomentosa were longer than those of the wild type plant, and the lateral roots were well developed. At the same time, fresh weight of roots of transgenic P. tomentosa was higher than that of the wild type plant (Table 2). 
To regulate the absorption rate of mineral nutrients and ensure the normal activities of plants under P-deficient conditions, the expression levels of $\mathrm{P}$ transporters and various other related genes are increased (Abel et al. 2010). For example, members of the $\mathrm{P}$ transporter family, PHT1 and PHT2 (Rae et al. 2003; Catarecha et al. 2007; Wu et al. 2011); the transcription factors, PHRI and PHR2 (Jie et al. 2008; Ren et al. 2012); and PHO1 and PHO2 involved in $\mathrm{P}$ transport (Franco-zorrilla et al. 2004; Bari et al. 2006; Stefanovic et al. 2011) are induced by low-P stress. The present study investigated the growth status and related gene expression of transgenic and wild type $P$. tomentosa under P-deficient conditions. Under the influence of OSPSTOL1 overexpression, the levels of PHT1, PHR1, PHR1, and PHOl expression in transgenic plants were higher than those in wild type plants. The expression of these genes has been shown to increase the soil nutrient absorption of transgenic $P$. tomentosa. In summary, the present study provides a theoretical basis for improving the absorption and utilization of $\mathrm{P}$ by $P$. tomentosa and other woody plants.

\section{Conclusion}

GUS staining and observation of the root indicated that OSPSTOL1 is expressed in the vegetative organs of the plants. In the case of poor nutritional status, transgenic plants had more developed roots to compensate for lower nutrient concentrations. Introduction of P-deficient conditions demonstrated that OSPSTOL1 participates in tolerance to $\mathrm{P}$ deficiency in $P$. tomentosa and can alleviate the associated symptoms. OsPSTOL1 can regulate the total content of N, P, and $\mathrm{K}$ and may be involved in the expression of genes related to the regulation of $\mathrm{P}$ uptake to alleviate the damage resulting from $\mathrm{P}$ deficiency stress in $P$. tomentosa.

\section{Acknowledgments}

This work was supported by the Guizhou Provincial Tea Industry Technology Innovation Center [Qianke Zhongyindi [2017] 4005], key technology research on tea quality improvement of albino, yellowed, and purple tea varieties [Qiankehe Platform Talents [2019] 5651], and the Guizhou Tea Industry System - Tea Tree Nutrition and Cultivation Function Experiment [Z184084].

\section{Author Contribution}

LLT conceived and designed the research, WL and YY conducted the experiments. WL contributed analytical tools and analyzed data. YXZ wrote the manuscript. YXZ and ZDG supervised the studies and revised the manuscript. All authors read and approved the manuscript.

\section{References}

Abel S, CA Ticconi, CA Delatorre (2010). Phosphate sensing in higher plants. Physiol Plantarum 115:1-8
Bari R, PB Datt, M Stitt, W Scheible (2006). PHO2, microRNA 399 and PHR1 define a phosphate-signaling pathway in plants. Plant Physiol 141:988-999

Catarecha P, MD Segura, JM Franco-Zorrilla, B García-Ponce, M Lanza, R Solano, J Paz-Ares, A Leyva (2007). A mutant of the Arabidopsis phosphate transporter PHT1;1 displays enhanced arsenic accumulation. Plant Cell 19:1123-1133

Ceasar SA (2018). Genome-wide identification and in silico analysis of PHT1 family genes and proteins in Setaria viridis: The best model to study nutrient transport in millets. Plant Genomics 12; Article 180019

Cheng LY, B Bucciarelli, JB Shen (2011). Update on white lupin cluster root acclimation to phosphorus deficiency. Plant Physiol 156:1025-1032

Chin, JH, R Gamuyao, C Dalid, M Bustamam, J Prasetiyono, S Moeljopawiro, M Wissuwa, S Heuer (2011). Developing rice with high yield under phosphorus deficiency: Pup1 sequence to application. Plant Physiol 156:1202-1216

Chin JH, X Lu, SM Haefele, R Gamuyao, A Ismail, M Wissuwa, S Heuer (2010). Erratum to: Development and application of gene-based markers for the major rice QTL Phosphorus uptake 1. Theor Appl Genet 120:1087-1088

Dai TT (2018). Effects of overexpression of AtBAS1 gene on the growth and development of Populus tomentosa. Master Thesis, p:33. Guizhou University, Guizhou, China

Franco-zorrilla JM, E Gonzalez, R Bustos, F Linhares, A Leyva, J Paz-Ares (2004). The transcriptional control of plant responses to phosphate limitation. J Exp Bot 55:285-293

Gamuyao R, JH Chin, J Pariascatanaka, P Pesaresi, S Catausan, C Dalid, I Slametloedin, EM Tecsonmendoza, M Wissuwa, S Heuer (2012). The protein kinase Pstoll from traditional rice confers tolerance of phosphorus deficiency. Nature 488:535-539

Gaxiola RA, M Edwards, JJ Elser (2011). A transgenic approach to enhance phosphorus use efficiency in crops as part of a comprehensive strategy for sustainable agriculture. Chemosphere 84:840-845

Heuer, X, X Lu, JH Chin, JP Tanaka, H Kanamori, T Matsumoto, TD Leon, VJ Ulat, AM Ismail, M Yano, M Wissuwa (2009). Comparative sequence analyses of the major quantitative trait locus phosphorus uptake 1 (Pupl) reveal a complex genetic structure. Plant Biotechnol J 7:456-471

Irfan M, M Abbas, JA Shah, MA Akram, MY Memon (2020). Categorization and identification of Brassica genotypes for phosphorus utilization efficiency. Intl J Agric Biol 23:227-234

Jewell MC, BC Campbell, ID Godwin (2010). Transgenic plants for abiotic stress resistance. In: Transgenic Crop Plants, pp:67-132. Springer, Berlin, Heidelberg

Jia XL, TX Wen, MX Gao, W Yang (2017). Leaf nitrogen and phosphorus concentration and the empirical regulations in dominant woody plants of shrublands across southern China. Chin J Plant Ecol 41:31-42

Jie Z, CJ Fang, CW Zhong, YL Yi, MW Xu, WH Xiao, QZ Wei, W Ping (2008). OsPHR2 is involved in phosphate-starvation signaling and excessive phosphate accumulation in shoots of plants. Plant Physiol 146:1673-1686

Liang Q, H Liao, X Yan (2007). Quantitative Analysis of Plant Root Architecture. Chin Bull Bot 24:695-702

Maharajan T, SA Ceasar, TPA krishna, M Ramakrishnan, V Duraipandiyan, AD Naif, A Bdulla, S Ignacimuthu (2018). Utilization of molecular markers for improving the phosphorus efficiency in crop plants. Plant Breed 137:10-26

MilnerMJ, RM Howells, C Melanie, B Sarah, G Neil, EJ Wallington (2018). A PSTOL-like gene, TaPSTOL, controls a number of agronomically important traits in wheat. BMC Plant Biol 18; Article 15

Ni JP, DS Wand (1998). Mapping QTLs for phosphorus deficiency tolerance in rice (Oryza sativa L.). Theor Appl Genet 97:1361-1369

Pariasca-Tanaka J, JH Chin, ND Khady, C Dalid, S Heuer, M Wissuwa (2014). A novel allele of the P-starvation tolerance gene OSPSTOL1 from African rice (Oryza glaberrima Steud) and its distribution in the genus Oryza. Theor Appl Genet 127:1387-1398

Qizhen ZM, TY Xi, M Lei (2008). The ecological roles and influencing factors of plant root architecture. Henan Sci 26:172-176 
Lu et al. / Intl J Agric Biol, Vol 25, No 3, 2021

Rae AL, DH Cybinski, JM Jarmey (2003). Characterization of two phosphate transporters from barley; evidence for diverse function and kinetic properties among members of the Pht1 family. Plant Molecular Biol 53:27-36

Rao I, M Pessarakli (1996). The role of phosphorus in photosynthesis. In: Handbook Photosynthesis, pp:173-194. Marcel Dekker, Inc. New York, USA

Ren F, QQ Guo, LL Chang, L Chen, CZ Zhao, H Zhong, XB Li (2012). Brassica napus PHRlGene encoding a MYB-Like protein functions in response to phosphate starvation. PLoS One 7; Article e44005

Sánchez-Calderón L, J López-Bucio, A Chacón-López, A Cruz-Ramírez, F Nieto-Jacobo, JG Dubrovsky, L Herrera-Estrella (2005). Phosphate starvation induces a determinate developmental program in the roots of Arabidopsis thaliana. Plant Cell Physiol 46:174-184

Schünmann PHD, AE Richardson, FW Smith, E Delhaize (2004). Characterization of promoter expression patterns derived from the Pht1 phosphate transporter genes of barley (Hordeum vulgare L.). J Exp Bot 398:855-865

Sheng XL (1999). The current state and prospect of plant nutrition and fertilizer science. Plant Nutr Fert Sci 5:193-205

SmithSN(1934). Response of inbred lines and crosses in maize to variations of nitrogen and phosphorus supplied as nutrients 1. Agron J 26:785-804

Stefanovic A, AB Arpat, R Bligny, E Gout, C Vidoudez, M Bensimon, Y Poirier (2011). Over-expression of PHO1 in Arabidopsis leaves reveals its role in mediating phosphate efflux. Plant J 66:689-699
Tong AHY, M Evangelista, AB Parsons, H Xu, GD Bader, N Page, M Robinson, S Raghibizadeh, CWV Hogue, H Bussey, B Andrews, M Tyers, C Boone (2001). Systematic genetic analysis with ordered arrays of yeast deletion mutants. Science 294:2364-2368

Wasaki J, T Shinano, K Onishi, R Yonetani, J Yazaki, F Fujii, K Shimbo, M Ishikawa, Z Shimatani, Y Nagata (2006). Transcriptomic analysis indicates putative metabolic changes caused by manipulation of phosphorus availability in rice leaves. J Exp Bot 57:2049-2059

Wu Z, H Ren, SP Mcgrath, P Wu, FJ Zhao (2011). Investigating the contribution of the phosphate transport pathway to arsenic accumulation in rice. Plant Physiol 157:498-508

Wu ZJ, C Tian, Q Jiang, XH Li, J Zhuang (2016). Selection of suitable reference genes for qRT-PCR normalization during leaf development and hormonal stimuli in tea plant (Camellia sinensis). Sci Rep 6; Article 19748

Xiang OY, H Xia, XQ Hong, X Zhao, W Zhang, X He, W Ma (2016). Knock out of the phosphate 2 gene TaPHO2-A1 improves phosphorus uptake and grain yield under low phosphorus conditions in common wheat. Sci Rep 6; Article 29850

Yao X, M Chen, D Zhao, L Lv (2020). Overexpression of the Sorghum bicolor $\mathrm{K}^{+} / \mathrm{Na}^{+}$transporter gene, $S b S K C 1$, enhances salt tolerance in poplar (Populus tomentosa). Intl J Agric Biol 24:304-310

Yu LP, CL Zhang, N Ma, J Li, SJ Zhang (2010). Rapeseed physiological response to drought and phosphorus deficiency. I. Gas exchange and yield. Chin J Oil Crop Sci 32:506-511 